\title{
Pandemic Influenza A (H1N1) 2009 and mortality in the United Kingdom: risk factors for death, April 2009 to March 2010
}

R G Pebody (Richard.Pebody@hpa.org.uk) ${ }^{1}$, E McLean $^{1}$, H Zhao ${ }^{1}$, P Cleary $^{2}$, S Bracebridge ${ }^{2}$, K Foster $^{2}$, A Charlett ${ }^{1}$, P Hardelid ${ }^{1}$,

P Waight ${ }^{1}$, J Ellis ${ }^{1}$, A Bermingham ${ }^{1}$, M Zambon ${ }^{1}$, B Evans ${ }^{1}$, R Salmon ${ }^{3}$, J McMenamin ${ }^{4}$, B Smyth ${ }^{5}$, M Catchpole ${ }^{1}$, J M Watson ${ }^{1}$

1. Health Protection Agency Centre for Infections

2. Health Protection Agency Local and Regional Services

3. National Public Health Service for Wales

4. Health Protection Scotland

5. Public Health Agency Northern Ireland

Citation style for this article:

Citation style for this article: Pebody RG, McLean E, Zhao H, Cleary P, Bracebridge S, Foster K, Charlett A, Hardelid P, Waight P, Ellis J, Bermingham A, Zambon M,

Evans B, Salmon R, McMenamin J, Smyth B, Catchpole M, Watson JM. Pandemic Influenza A (H1N1) 2009 and mortality in the United Kingdom: risk factors for death, April 2009 to March 2010. Euro Surveill. 2010;15(20):pii=19571. Available online: http://www.eurosurveillance.org/ViewArticle.aspx?Articleld=19571

This paper describes the epidemiology of fatal pandemic influenza $\mathrm{A}\left(\mathrm{H}_{1} \mathrm{~N}_{1}\right)$ cases in the United Kingdom (UK) since April 2009 and in particular risk factors associated with death. A fatal case was defined as a UK resident who died between 27 April 2009 and 12 March 2010, in whom pandemic influenza $A\left(\mathrm{H}_{1} \mathrm{~N}_{1}\right)$ infection was laboratory-confirmed or recorded on the death certificate. Case fatality ratios (CFR) were calculated using an estimated cumulative number of clinical cases as the denominator. The relative risk of death was estimated by comparing the population mortality rate in each risk group, with those not in a risk group. Across the UK, 440 fatal cases were identified. In England, fatal cases were mainly seen in young adults (median age 43 years, $85 \%$ under 65 years), unlike for seasonal influenza. The majority $(77 \%)$ of cases for whom data were available $(n=308)$ had underlying risk factors for severe disease. The CFR in those aged 65 years or over was nine per 1,000 clinical cases (range 3-26) compared to 0.4 (range 0.2 to 0.9 ) for those aged six months to 64 years. In the age group between six month and 64 years, the relative risk for fatal illness for those in a risk group was 18 . The population attributable fractions in this age group were highest for chronic neurological disease (24\%), immunosuppression $(16 \%)$ and respiratory disease $(15 \%)$. The results highlight the importance of early targeted effective intervention programmes.

\section{Introduction}

Seasonal influenza is responsible for excess winter all-cause mortality and hospitalisations - particularly amongst the elderly, the very young and those with underlying high-risk conditions such as chronic heart and lung disease [1]. The extent of any observed excess mortality varies each year and depends upon various factors including the predominant circulating influenza strain [2].
Since the emergence of pandemic influenza $A\left(\mathrm{H}_{1} \mathrm{~N}_{1}\right)$ in April 2009 and initial concerns about the risk of severe respiratory illness, much effort has been devoted to rapidly understanding the severity and impact of this novel influenza virus. Early work in various settings has demonstrated that the overall case fatality ratio (CFR) is generally low [3-5], with similar risk factors for severe disease as seasonal influenza, although in addition, individuals who are obese or pregnant are reportedly at high risk of severe outcome [6-8].

By 12 March 2010, more than 400 deaths had been reported across the United Kingdom (UK) by the Chief Medical Officer and health protection organisations in Wales, Scotland and Northern Ireland [9]. Information on which groups are at higher risk of death is key to healthcare planning - in particular the development and evaluation of the pandemic influenza $A\left(\mathrm{H}_{1} \mathrm{~N}_{1}\right)$ vaccination programme. The vaccination programme in the UK started in October 2009 and initially targeted individuals at higher risk of severe disease, including pregnant women [10]. In December 2010, vaccination for all children from six month to five years of age began.

Using surveillance data collected during the pandemic in the UK, this paper aims to describe the epidemiology of fatal pandemic influenza $A\left(\mathrm{H}_{1} \mathrm{~N}_{1}\right)$ cases. It sets out to estimate various mortality indicators by age and clinical risk group to inform the implementation of prevention and control programmes for pandemic influenza.

\section{Methods}

The methods initially outline the case definitions, how cases were ascertained and what additional epidemiological data, including risk factor information, was gathered. The descriptive section (time and place) covers the entire UK (England, Wales, Scotland and Northern Ireland). The analytical section (the 
population mortality rates, CFRs and population attributable fractions) only covers England.

\section{Case definition}

A fatal case was defined as a resident in the UK who died after 27 April 2009, in whom pandemic influenza $A\left(H_{1} N_{1}\right)$ virus infection was confirmed by a laboratory ante- or post-mortem (either by RT-PCR or serology) or by any mention on the death certificate and was reported up to 12 March 2010.

\section{Case ascertainment}

Fatal cases in England were ascertained by the Health Protection Agency (HPA) over this period from several different reporting sources: Firstly, local healthcare providers reported fatal pandemic influenza cases to HPA-run local Health Protection Units (HPUs). Secondly, the immunisation department of the HPA Centre for Infections followed up laboratory-confirmed pandemic influenza cases with general practitioners (GPs) as part of monitoring the pandemic influenza vaccination programme. This follow-up included ascertainment of outcome status. Thirdly, hospital clinicians reported confirmed hospitalised cases of pandemic influenza to a web-based hospital surveillance system for pandemic influenza $A\left(\mathrm{H}_{1} \mathrm{~N}_{1}\right)$ infection. This included ascertainment of outcome status. Fourthly, the Office for National Statistics (ONS) shared each week individual death registrations with the HPA Centre for Infections of any death with a mention of influenza (ICD-10 codes Jo9, J10 and J11).

Individual fatal cases were reconciled and de-duplicated using available personal identifiers. All fatal cases were verified as laboratory-confirmed by matching to laboratory records of confirmed pandemic influenza held by the HPA or by any mention of influenza on the death certificate from ONS. They were confirmed as deceased either through the NHS Patient Demographic Service or through death certificate from ONS. Deaths were also compared with fatal cases collected by the Office of the Chief Medical Officer in England [3].

Fatal cases outside England were identified by the Boards of Health by Health Protection Scotland and through their equivalents in Wales and Northern Ireland.

The work was carried out under NHS Act 2006 (section 251), which provides statutory support for disclosure of such data by the NHS, and their processing by the HPA, for purposes of communicable disease control. Ethical approval was not required and informed consent was not sought.

\section{Case follow-up}

Clinical and demographic data and information on underlying risk conditions were gathered from the local HPU, the hospital physician or the general practitioner using a standard questionnaire. For those fatal cases for whom such data were not available, risk factor information was extracted from the death certificate, if available.

\section{Risk factor definition}

The seasonal influenza risk groups used throughout this paper were those defined by the UK Department of Health (DH) for the seasonal influenza vaccination programme in 2008-9: chronic respiratory disease, including asthma treated in the last three years; chronic heart disease, chronic liver disease, chronic renal disease, chronic neurological disease, stroke/transient ischaemic attack, and immunosuppression through disease or treatment and diabetes.

The risk groups for pandemic influenza in addition included pregnancy and obesity, which are presented separately.

\section{Population mortality rates}

Age-specific population mortality rates for pandemic influenza were calculated using the mid-2008 population for England (Source: ONS).

\section{Seasonal influenza deaths}

Death registrations between 2 January 2001 and 2 February 2009 with an ICD-10 code for influenza (ICD$10 \mathrm{~J} 09, \mathrm{~J}_{10}$ and J11) in England were also identified to compare the epidemiology of fatal pandemic influenza with fatal seasonal influenza. Information on whether the person suffered from underlying risk conditions was not specifically gathered for these fatal cases; deaths were classified into DH standard risk groups as above, based on available text information recorded for cause of death on the certificate. The distribution of $\mathrm{DH}$-defined risk groups for severe disease amongst deaths with laboratory-confirmed pandemic influenza $A\left(\mathrm{H}_{1} \mathrm{~N}_{1}\right)$ and deaths with any mention of seasonal influenza were compared using age-adjusted MantelHaenszel odds ratios (OR). To calculate the population mortality rate for seasonal influenza deaths, the denominators were the cumulative mid-year annual population estimates for England for the period from 2001 to 2008 (source: ONS).

\section{Population prevalence of specific}

risk factors in England

The prevalence of specific high risk conditions (excluding pregnancy and obesity) in the English population were derived from the DH influenza vaccine uptake monitoring system [11]. All GPs in England are requested to extract data on registered patients from their health information systems each season using standard queries. This system was used to determine the total number of people registered with a general practice in England and the number of those older than six months eligible for seasonal influenza vaccine by age group and by individual risk group. These risk groups did not include pregnancy or obesity, as these are not DH-defined target groups for the seasonal influenza vaccination programme [11]. For people aged between six months and 64 years a breakdown by individual 
DH-defined seasonal influenza risk group was available (based on data provided by $96.2 \%$ of all English GP practices) in two age groups: six months to 15 years and 16 years and over [12]. For those aged 65 years and over information on the number in any $\mathrm{DH}$-defined risk group was available and was extrapolated from data provided by $79.4 \%$ of GP practices (provisional data provided by the $\mathrm{DH}$ ). Using this approach, as people may fall into more than one risk group, the sum of all the individual risk groups will exceed the total number in the population [11].

The point prevalence of pregnant women was calculated using the estimated English mid-2008 female population aged 15 to 44 years (source: ONS) as the denominator. According to ONS, an estimated 676,236 maternities (live and still births) occurred in England in 2008 [13]. Assuming $4 \%$ of the female population of child-bearing age (15 to 44 years) experience a miscarriage or abortion in any given year [14] and using the mid-2008 estimate of the female population aged 15 to 44 years $(10,532,500)$, an annual figure of 421,300 miscarriages/abortions was calculated. To calculate the number of women who were pregnant at any one time, 9/12 of the annual number of maternities (assuming these pregnancies have a duration of nine months) was added to $3 / 12$ of the annual number of miscarriages/ abortions (assuming these pregnancies have an average duration of three months). A final figure of 612,502 pregnancies ( $5.8 \%$ of the female population aged 15 to 44 years) was reached.

\section{Case fatality ratios for clinical}

\section{pandemic influenza in England}

The CFRs were calculated overall and by age and risk group. The numerator was fatal cases in England and the denominator was the estimated cumulative number of clinical cases in England from the beginning of the pandemic to 21 February 2010. This includes a three week lag. This lag incorporates the observed median delay from disease onset to report of death (see results section). The estimated number of clinical cases was calculated by the HPA using a statistical model which relies on data from various surveillance systems: the primary care-based QSurveillance scheme, sentinel virological surveillance schemes and data from the National Pandemic Flu Service (NPFS) [15].

The estimated number of clinical cases consulting health services by age group were calculated using age group-specific data from the various surveillance systems (available in the following age groups: <1 year, 1-4, $5-14,15-24,25-44,45-64$ years and $\geq 65$ years). To estimate the number of clinical cases in each DH-defined risk group, the proportion of people aged between six months and 64 years in each risk group from the GP vaccine uptake survey described above was applied to the cumulative number of cases estimated by the HPA in the same age group. This assumes that those in a clinical risk group are as likely to have symptomatic infection as those not in a clinical risk group.
It was assumed that babies aged under six months represent half the infant (aged under one year) clinical cases. To estimate the number of pregnant clinical cases, the point prevalence of pregnancy (as described above) was applied to half of the estimated number of clinical cases aged 15 to 44 years (assuming an equal distribution of influenza infection between males and females).

The HPA's estimate of clinical case numbers is subject to uncertainties particularly regarding consultation behaviour. A lower and upper estimate was calculated to allow for this. A sensitivity analysis was undertaken: the overall and the age- and risk group-specific CFRs were calculated using the central estimate for the number of clinical cases, and for the low and high estimates. A 95\% exact binomial confidence interval (Cl) was calculated around each case fatality estimate. The range incorporating the $95 \% \mathrm{Cl}$ around the CFRs calculated from the low and high estimated number of clinical cases is presented.

\section{Relative risk of fatal pandemic influenza in England}

The relative risk (RR) of fatal pandemic influenza for each risk group was calculated by comparing the population mortality rate in each individual risk group with the rate in those who did not fall into any risk group. For those aged under 65 years, Mantel-Haenszel ageadjusted RR, with corresponding exact $95 \% \mathrm{Cl}$ were calculated for each risk group using the two available age groups (from six months up to 15 years and from 16 to 64 years). For those aged 65 years and over, information was only available overall for any risk group to calculate RR of fatal infection.

\section{Population attributable fractions for fatal pandemic influenza in England}

The population attributable fraction (PAF) of each individual risk group to fatal pandemic influenza was estimated. The PAF was calculated by dividing the difference in the overall population mortality rate (PMR) and the PMR in the non-exposed by the overall PMR. The unexposed group were people without that particular risk factor (i.e. all other cases, who may fall into other risk groups), which is different to that used to calculate the RR (people with no risk factor at all). The PAF takes into account the prevalence of the exposure in the population and is interpreted as the proportion of the total number of deaths which would be averted, if the exposure were completely removed.

\section{Laboratory methods}

Laboratory confirmation of pandemic influenza $A\left(\mathrm{H}_{1} \mathrm{~N}_{1}\right)$ virus was performed using respiratory swabs collected into virus transport medium. All samples were tested either at the Respiratory Virus Unit of the HPA Centre for Infections, London or at the HPA Regional Microbiology Network Laboratories, using real-time RT-PCR assays for detection of influenza $A$, and subtyped for pandemic influenza $A\left(\mathrm{H}_{1} \mathrm{~N}_{1}\right)$ viruses $[16,17]$ 
Number of deaths from confirmed pandemic influenza A(H1N1) by week and country of death, June 2009-February 2010 $(n=436$, date of death missing in four cases) with estimated number of clinical cases in England (HPA)

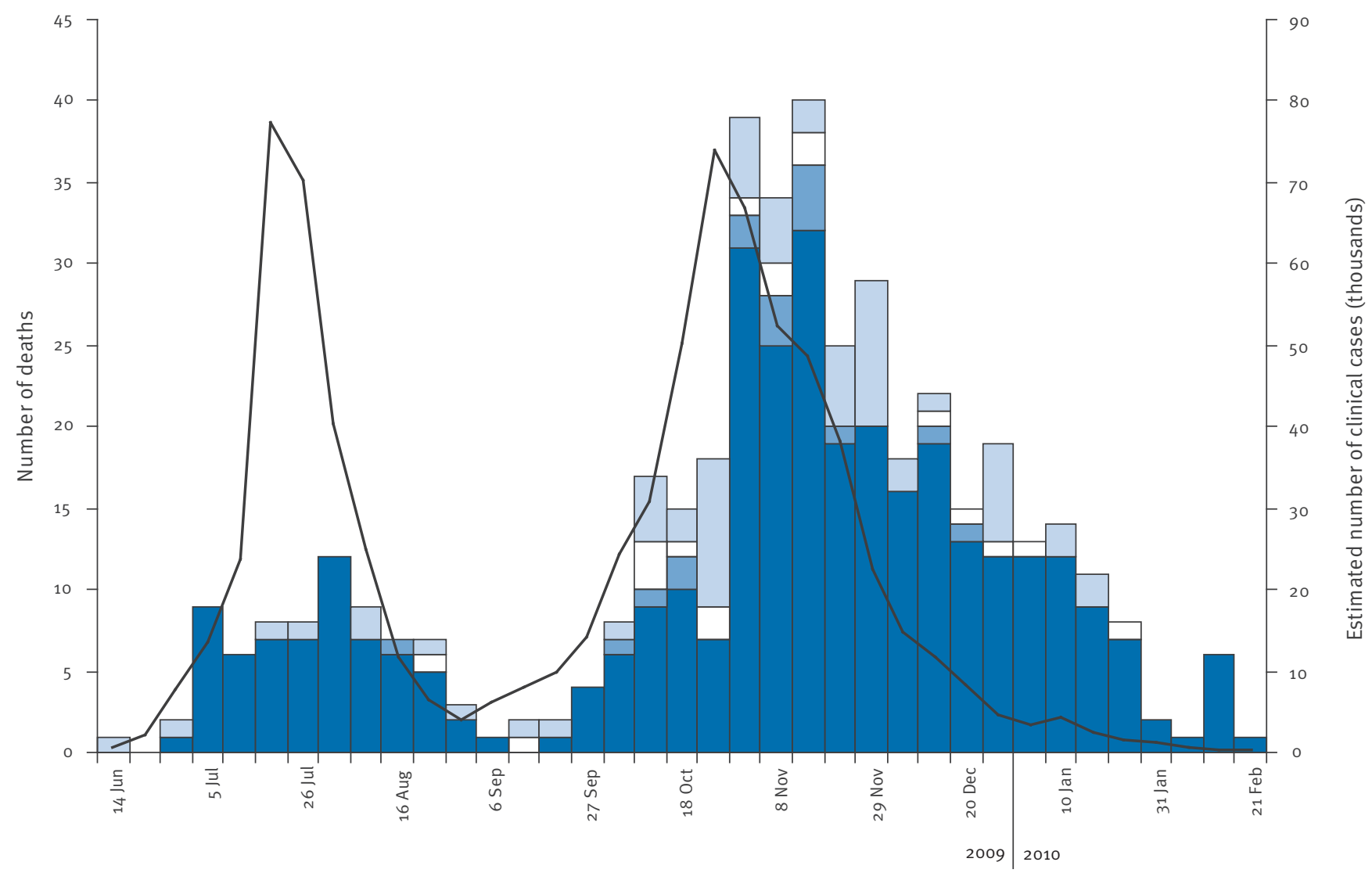

Week (ending date)

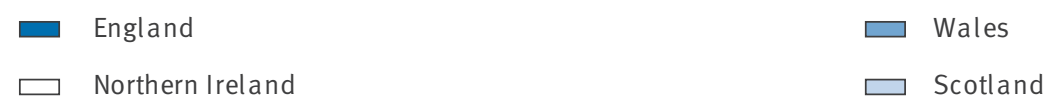

Estimated number of clinical cases

\section{FIGURE 2}

Age distribution of (A) pandemic influenza deaths, England, until February $2010\left(\mathrm{n}=336^{\mathrm{a}}\right)$, with cumulative population pandemic influenza mortality rate, and (B) seasonal influenza deaths, England, January 2001-February 2009) (n=334), with average annual population mortality rate (2001-2008)

A

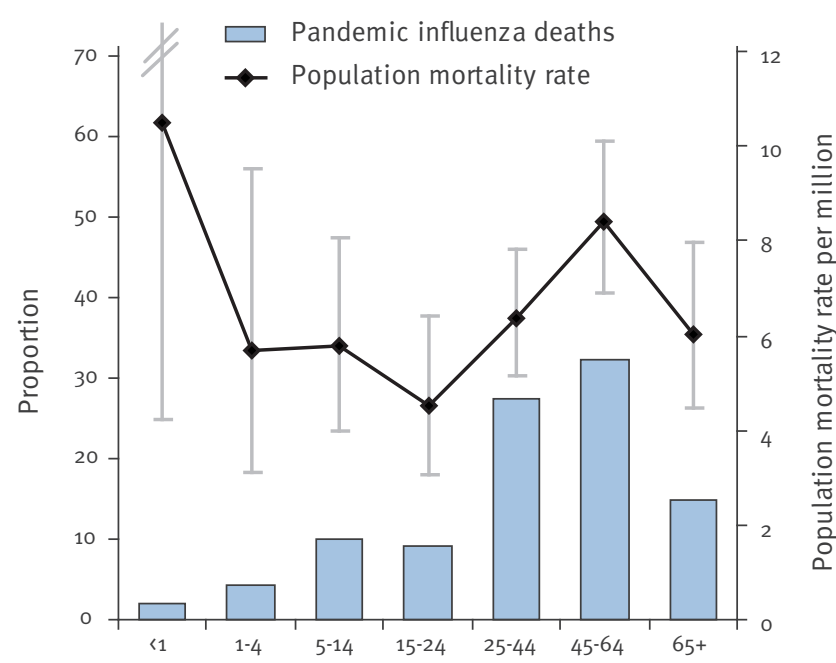

Age group (years)
B

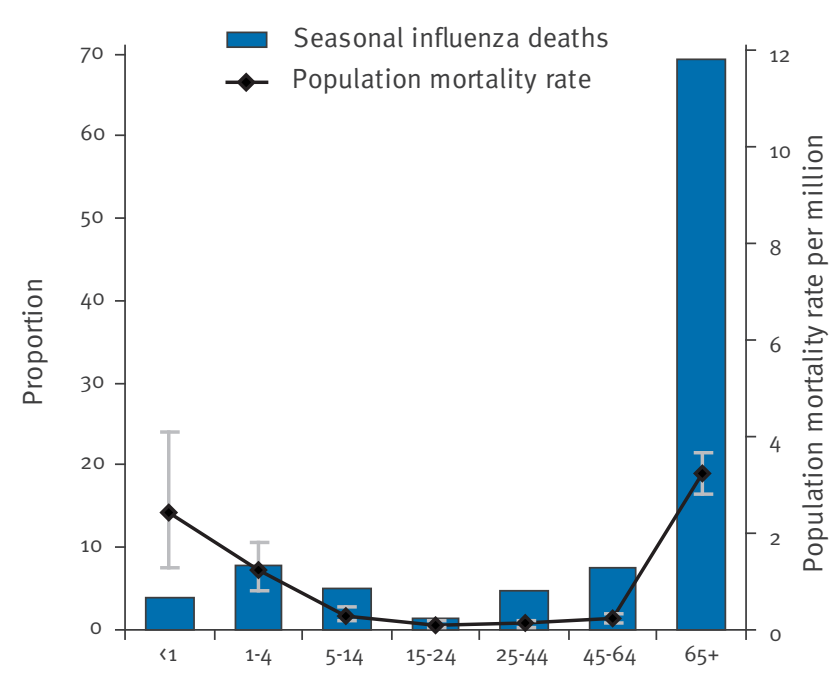

Age group (years)

${ }^{a}$ Age not available for one fatal pandemic influenza case. 


\section{Results}

Fatal pandemic influenza cases by time and place in the United Kingdom

Individual information on 440 fatal pandemic influenza cases fulfilling the case definition was collated by the HPA from across the UK up to 12 March 2010: 337 deaths occurred in England, 65 in Scotland, 21 in Northern Ireland and 17 in Wales. Of the 440 deaths, $387(88 \%)$ were laboratory-confirmed and 53 were only confirmed by mention of influenza on the death certificate.

The first reported death occurred in the week ending on 14 June 2009 (Figure 1). The number of deaths by date of death climbed to a peak in the week ending on 2 August, with the onset of the school summer holidays and declined to baseline levels by the beginning of September. This pattern reflects the summer pandemic wave as illustrated by the weekly estimated number of clinical cases of pandemic influenza in England. A small number of deaths continued to be reported each week throughout September, followed by an increase from October onwards coinciding with a second increase in pandemic influenza activity in the autumn. This second larger peak of deaths occurred in mid-November and decreased to low levels by the end of January 2010. The final reported death included in this analysis occurred in the week ending on 21 February 2010. Seventy-three $(17 \%)$ of the 440 fatal cases in the UK occurred during the summer wave (until end August 2009) and 367
$(83 \%)$ during the autumn/winter wave (from the beginning of September 2009 to February 2010).

\section{Age distribution of fatal pandemic and seasonal influenza cases in England} Of the 336 pandemic fatal cases in England with information on age, the median age of fatal pandemic cases was 43 years (interquartile range (IQR): 24-57) and the mean was 41 years (standard deviation (SD): 22). The population mortality rate from pandemic influenza was higher in the under one-year-olds than in other age groups (Figure 2A). Only four (1\%) of 336 fatal pandemic cases were reported in children under six months of age. Population mortality rates were not elevated in 1-4 year olds compared to older age-groups.

A total of 334 fatal seasonal influenza cases were collated from death certificates for the period between 2 January 2001 and 2 February 2009 in England. The seasonal influenza deaths occurred mainly in people aged 65 years or over (Figure 2B), with 232 (69\%) of 334 seasonal influenza deaths compared to $50(15 \%)$ of 336 pandemic influenza deaths (Fisher's exact test, p $<0.0001)$. There was no evidence of a significant difference in the proportion of deaths in children younger than six months from pandemic influenza compared to seasonal influenza (Fisher's exact test, $p=0.24$ ). The average age was higher in fatal seasonal influenza cases compared to fatal pandemic influenza, with

\section{FIGURE 3}

Distribution of pandemic influenza A(H1N1) deaths (2009-2010, n=308 $)$ and seasonal influenza deaths (2001-2009, $\mathrm{n}=334)$ by reported underlying risk factor, England

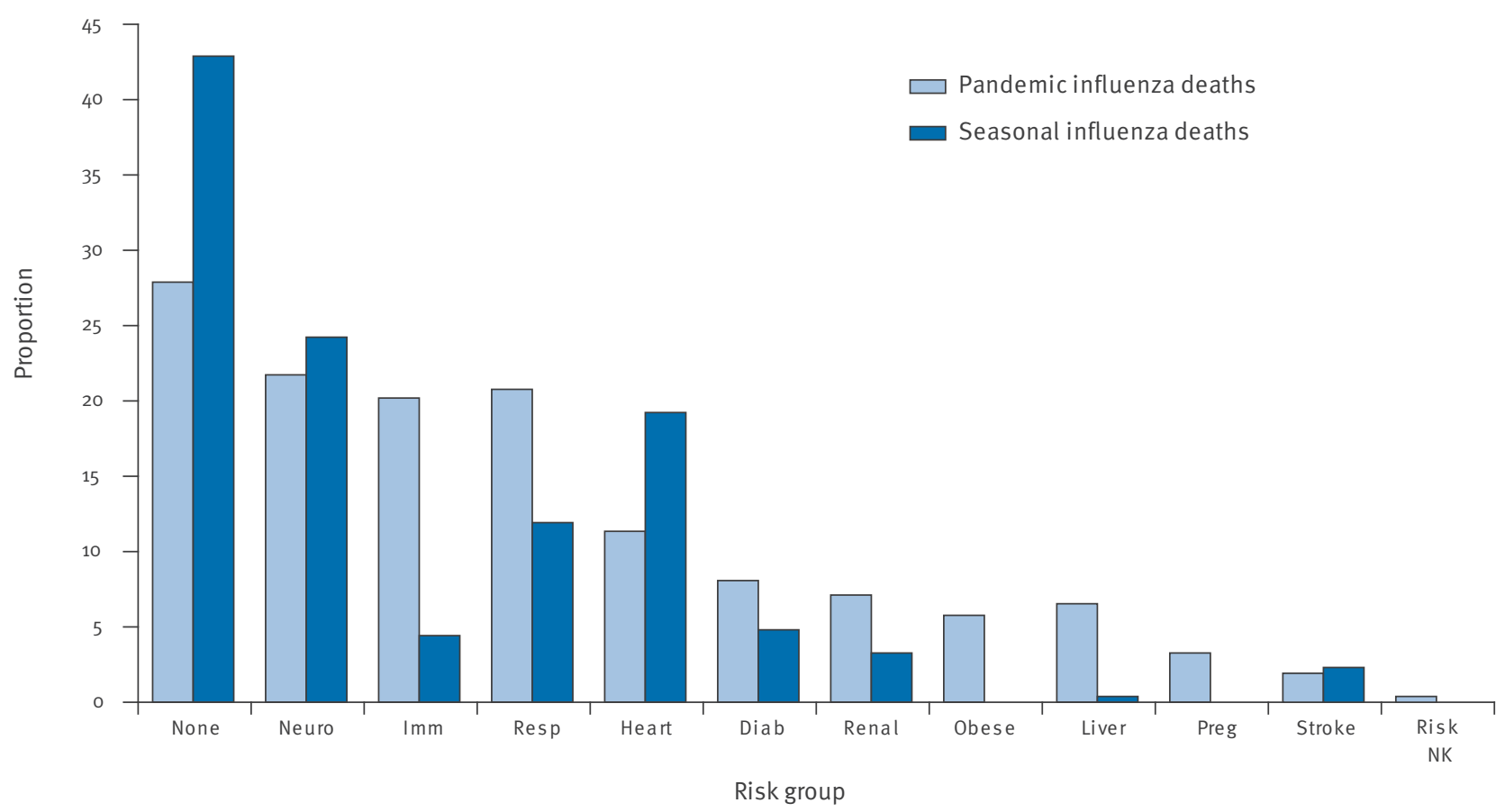

Risk groups: chronic neurological disease (Neuro), chronic respiratory disease including asthma (Resp), immunosuppression through disease or treatment (Imm), chronic heart disease (Heart), diabetes (Diab), chronic renal disease (Renal), obesity (Obese), chronic liver disease (Liver), pregnancy (Preg) and stroke/transient ischaemic attack (Stroke), no risk group including pregnancy and obesity (None).

Cases may fall into more than one risk group.

a Excluding cases with no available information on risk group. 
a median of 81 years (IQR: $53-89$ ) and a mean of 66 years (SD: 32 ).

\section{Distribution of underlying risk factors} amongst pandemic influenza and seasonal influenza fatal cases in England

Data on risk group were missing for 29 (9\%) of 337 cases. Information was more likely to be missing in young children $(p=0.004,36 \%$ in the 1 -4-year-olds

\section{TABLE 1}

Distribution of risk factors for fatal pandemic influenza A(H1N1), England only, April 2009-February 2010 $(\mathrm{n}=308)$

\begin{tabular}{|c|c|}
\hline Underlying risk factor & Total $\left(\%{ }^{a}\right)$ \\
\hline Chronic neurological disease & $67(22 \%)$ \\
\hline Cerebral palsy/developmental delay & 22 \\
\hline Neuro-musculoskeletal disorders & 10 \\
\hline Down's syndrome & 7 \\
\hline Epilepsy & 9 \\
\hline Chronic respiratory disease & $63(20 \%)$ \\
\hline Chronic obstructive pulmonary disease & 20 \\
\hline Asthma & 19 \\
\hline Immunosuppression & $62(20 \%)$ \\
\hline Leukaemia/lymphoma & 20 \\
\hline Myeloma & 14 \\
\hline Solid tumour & 8 \\
\hline Organ/bone marrow transplant & 9 \\
\hline Chronic heart disease & 35 (11\%) \\
\hline Congenital & 9 \\
\hline Diabetes & $25(8 \%)$ \\
\hline Chronic renal disease & $22(7 \%)$ \\
\hline Chronic liver disease & $20(6 \%)$ \\
\hline Alcohol-related & 12 \\
\hline Obesity & $18(6 \%)$ \\
\hline Pregnancy & $10(3 \%)$ \\
\hline
\end{tabular}

Cases may fall into more than one risk group and suffer from more than one disorder within each risk group.

a Proportion of all cases with known risk group status $(n=308)$. compared to all other age groups in the range of $0-15 \%)$ and in the autumn wave $(p=0.028,2 \%$ in the summer wave versus $10 \%$ in the autumn wave). Of the 308 fatal pandemic influenza cases in England with available data, 222 (72\%) fatal cases had an underlying risk factor for severe influenza and fell into DH-defined risk groups recommended for the 2008-9 seasonal influenza vaccine (excluding pregnancy and obesity). Twenty-eight people had these latter underlying conditions, 15 of whom (nine obese and six pregnant fatal cases) did not have any other underlying risk factor. Including these 15 , this gives a total of $77 \%$ (237 of 308) of fatal pandemic influenza cases in a risk group. The majority $(n=171,72 \%)$ of the 237 fatal cases with an underlying risk factor had only one risk factor, however, 43 fatal cases suffered from two underlying conditions, 20 from three, and three from four or five risk factors.

The distribution of fatal cases of pandemic influenza by reported underlying risk factor is shown in Figure 3. Chronic neurological disease, respiratory disease and immunosuppression were the most common reported risk factors. A similar pattern in the distribution of individual risk factors was observed for seasonal influenza deaths, although, after adjusting for age, fatal cases of pandemic influenza were more likely to be associated with a risk factor than fatal cases of seasonal influenza (age-adjusted OR: 2.8; $95 \% \mathrm{Cl}$ 1.7-4.5; pro.0001). For fatal seasonal influenza cases, $57 \%$ (191 of 334) had an underlying risk factor (excluding pregnancy and obesity). The most common risk factors were chronic neurological and cardiac disease (Figure 3). There was evidence that immunosuppression (age-adjusted OR: 4.3; $95 \% \mathrm{Cl}: 2.4-7.8)$ and chronic renal disease (ageadjusted OR: $2.7 ; 95 \% \mathrm{Cl}: 1.2-6.2$ ) were more common in pandemic influenza deaths than seasonal deaths.

The distribution of individual risk factors in the English pandemic fatal cases is shown in Table 1 . The most commonly reported underlying risk factor in children (aged under 16 years) was neurological disease,

\section{TABLE 2}

Proportion of fatal pandemic $(n=308 a)$ and seasonal $(2001-2009, n=334)$ influenza cases with risk factors compared to the general population, by age group, England

\begin{tabular}{|c|c|c|c|c|c|c|c|c|c|c|c|}
\hline \multirow[b]{2}{*}{ Age group } & \multicolumn{3}{|c|}{ Population } & \multicolumn{4}{|c|}{$\begin{array}{l}\text { Pandemic influenza deaths (April } \\
\text { 2009-February 2010) }\end{array}$} & \multicolumn{4}{|c|}{ Seasonal influenza deaths (2001-2009) } \\
\hline & Risk group & Total & $\%$ & Risk group & Total & $\%$ & $\mathbf{p}^{\mathbf{b}}$ & Risk group & Total & $\%$ & $\mathbf{p}^{\mathbf{b}}$ \\
\hline$<6$ months & $\mathrm{N} / \mathrm{A}$ & 333,800 & $\mathrm{~N} / \mathrm{A}$ & 4 & 4 & $100.0 \%$ & $\mathrm{~N} / \mathrm{A}$ & 4 & 8 & $50.0 \%$ & $\mathrm{~N} / \mathrm{A}$ \\
\hline $\begin{array}{l}\ll 6 \text { months - } \\
15 \text { years }\end{array}$ & 535,933 & $9,225,079$ & $5.8 \%$ & 32 & 44 & $72.7 \%$ & $<0.0001$ & 28 & 49 & $57.1 \%$ & $<0.0001$ \\
\hline $16-64$ years & $4,043,312$ & $34,724,600$ & $11.6 \%$ & 145 & 215 & $67.4 \%$ & $<0.0001$ & 29 & 45 & $64.4 \%$ & $<0.0001$ \\
\hline $\begin{array}{l}65 \text { years } \\
\text { and over }\end{array}$ & $4,233,686$ & $8,285,300$ & $51.1 \%$ & 41 & 45 & $91.1 \%$ & $<0.0001$ & 130 & 232 & $56.0 \%$ & 0.148 \\
\hline Total & $8,812,931$ & $52,568,779$ & $16.8 \%$ & 222 & 308 & $72.1 \%$ & $<0.0001$ & 191 & 334 & $57.2 \%$ & $<0.0001$ \\
\hline
\end{tabular}

N/A: not applicable.

a Of those with known risk group status (excluding pregnancy and obesity).

${ }^{b} \mathrm{p}$ value for Fisher's exact test of difference in proportions. 


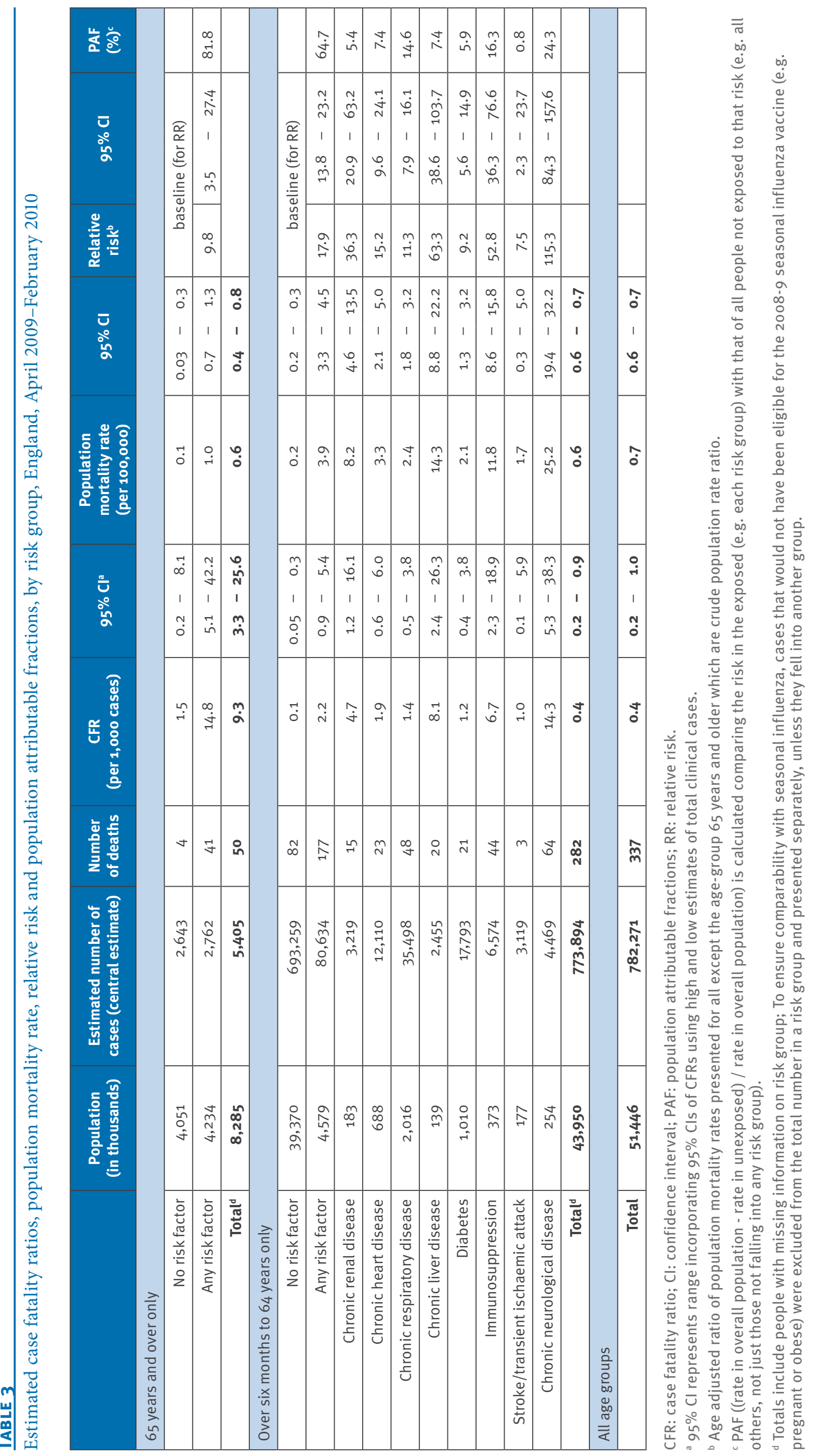


whereas in adults (aged 16 years or over), immunosuppression was the most common. There were also 71 other fatal cases that had no reported underlying risk factor for severe disease. Adults aged 25-44 years had the highest proportion without an underlying reported risk factor for severe disease $(28 / 87,32 \%)$.

The mean duration of time from illness onset to death amongst fatal pandemic influenza cases was 12 days, the median was 9.5 days (IQR: $5-17$, range: $0-45$ days) $(n=116$ on whom such information was known).

\section{Prevalence of underlying risk factors for} severe disease in the general population and in fatal cases of seasonal and pandemic influenza in England

The prevalence of any risk factor for severe disease in the general population of England between six months and 64 years of age was $10 \%$, whereas it was $51 \%$ for people aged 65 years or over (Table 2).

There was strong evidence in all age groups for a significant difference ( $p<0.0001$ ) between the people who died with pandemic influenza and the general population regarding the proportion with at least one risk factor (excluding obese and pregnant fatal cases). A similar pattern was seen for deaths due to seasonal influenza (Table 2).

\section{Case fatality ratios for pandemic influenza}

\section{A(H1N1) in England by risk factor}

The overall estimated symptomatic CFR was 0.4 per 1,000 clinical cases. The $95 \%$ confidence limits around CFR estimates, using low and high estimates for the cumulative number of clinical cases, gave a range of 0.2 to 1 per 1,000 clinical cases. There was evidence of differences in the age-specific CFRs, with a higher CFR in those over 65 years of age (CFR: 9; range: $3-26$ per 1,000 clinical cases) compared to those aged six months to 65 years (CFR: 0.4 ; range: $0.2-0.9$ per 1,000 clinical cases).

The estimated CFR for clinical cases aged between six months and 64 years in a risk group (excluding obese and pregnant cases) was 2 per 1,000 cases compared to 0.4 per 1,000 case for those not in a clinical risk group (Table 3). The CFR for clinical cases aged 65 years or over in a clinical risk group was estimated to be 15 per 1,000 clinical cases compared to 1.5 per 1,000 for those aged 65 years or over who were not in a risk group.

The estimated CFR also varied by individual risk group (Table 3). In particular a much higher CFR was observed amongst clinical cases with underlying immunosuppression, chronic liver disease or chronic neurological disease.

The CFR for pregnant women was 0.9 per 1,000 clinical cases ( $95 \% \mathrm{Cl}$ : $0.2-3.5$ per 1,000$)$.
Population mortality rate and relative risk of fatal illness in England by risk group

In the age group between six months and 64 years of age, the population mortality rate by risk group was highest for those with underlying chronic neurological disease, chronic liver disease and immunosuppression (Table 3). The population mortality ratio for pregnant women was 1.6 per 100,000 ( $95 \% \mathrm{Cl}$ : 0.8-3.0 per 100,000).

The RR of fatal illness from pandemic influenza $A\left(\mathrm{H}_{1} \mathrm{~N}_{1}\right)$ virus infection was highly elevated for those with a risk factor in all age groups (Table 3 ). In the age group of six months to 64 year-olds, the highest risk groupspecific age-adjusted RR of fatal illness was found in those with immunosuppression, chronic liver disease and chronic neurological disease.

The RR of fatal illness for pregnant women was also elevated (RR: 7; 95\% Cl: 3-15) compared with women of child-bearing age with no risk factor (15-44 years).

Population attributable fraction for fatal pandemic influenza in England The PAF was $82 \%$ for any risk factor in fatal cases aged 65 or over and $65 \%$ for those aged six months to 64 years. This means that $65 \%$ of the pandemic influenza deaths in the English population aged six months to 64 years could potentially be prevented by protecting all those in a clinical risk group.

The highest risk group-specific PAFs for those aged six months to 64 years were for chronic respiratory disease, immunosuppression and chronic neurological disease (Table 3).

\section{Discussion}

After the summer and autumn/winter waves of pandemic influenza $\left(\mathrm{H}_{1} \mathrm{~N}_{1}\right)$ 2009, more than 400 fatal cases had been reported across the UK. Unlike seasonal influenza, where deaths occur mainly amongst the elderly, the majority of the pandemic influenza deaths were among young and middle-aged adults. Most fatal pandemic influenza cases had underlying risk factors for severe illness and although the overall CFR has been low, it was significantly higher amongst those in clinical risk groups. The RR and population attributable fractions for fatal pandemic influenza were particularly high for groups with underlying chronic neurological disease, chronic respiratory disease, chronic liver disease and immunosuppression. Pandemic influenza in pregnancy has also been demonstrated to be a risk factor for death.

There are several limitations to this study. Firstly, the CFR estimates rely on estimated numbers of clinical cases consulting health services in the population. There is considerable uncertainty about these denominators. If the number of symptomatic cases has been underestimated, then the CFR will have been overestimated. An indication of the uncertainty is captured by 
including the upper and lower limits of the estimated number of cases in the CFR calculations. Secondly, fatal case ascertainment may be incomplete. To minimise this, data has been reconciled from several independent sources. Thirdly, data on risk factors are missing for $9 \%$ of pandemic fatal cases. Fourthly, we did not have information on trimester at time of death for pregnant women. Consequently, to derive our denominator of pregnant women we used an estimate for all pregnancies including those that had a miscarriage. If the risk of death is greatest in the later stages of pregnancy, this may have led to an under-estimation of the risk of fatal disease amongst pregnant women. Fifthly, the results of the comparison between seasonal and pandemic influenza should be interpreted cautiously, as the method of collection of risk group information in the two datasets was different. Finally, some of the cases had severe underlying medical conditions, with influenza as a contributing cause of death. We do not know what proportion of these deaths could have been prevented by protecting against influenza.

Several hundred fatal pandemic influenza $A\left(\mathrm{H}_{1} \mathrm{~N}_{1}\right)$ cases have been reported in the UK since spring 2009. Laboratory-confirmed fatal cases have been identified in all age groups, mainly amongst younger adults, with only a minority of deaths in those aged over 65 years. Although the population mortality rate for pandemic influenza virus infection was low in the elderly, the CFR of pandemic influenza in this group was high. A very different picture was apparent for seasonal influenza, where the majority of deaths based on death certificate analysis were in those in 65 years or older. This is illustrated by excess mortality at the population level during seasonal influenza seasons, with the excess normally occurring amongst the elderly [18]. The observation that few confirmed pandemic influenza deaths occurred amongst those aged 65 years or older suggests that this part of the population is at least partially protected from the infection. This concurs with cross-sectional seroprevalence data from the United States (US) and the UK, which show that a substantial proportion of the birth cohort born before 1957 have cross-reactive antibodies to the pandemic influenza $A\left(\mathrm{H}_{1} \mathrm{~N}_{1}\right)$ virus $[19,20]$. This would be consistent with exposure to influenza $A\left(\mathrm{H}_{1} \mathrm{~N}_{1}\right)$ viruses circulating in the population in the period from 1918 to 1957 and explains the relatively low disease burden in these older cohorts during the present pandemic.

We report an overall CFR of 0.4 per 1,000 cases of pandemic influenza. This CFR is consistent with earlier published work from the UK by Donaldson et al. [3], but also from the US [5] and from reports from the southern hemisphere [21]. This figure also fits with the clinical picture associated with pandemic influenza virus infection that continues to be a generally mild disease for most cases. The observed CFR is much lower than observed in previous pandemics [3]. This low case fatality is in agreement with data from the routine excess mortality surveillance system operating in England and Wales, where no excess all-cause mortality was observed until the end of December 2009 either overall or in any individual age group based on daily and weekly mortality returns from the General Registry Office and the ONS. However, a small excess in all-cause mortality was observed in weeks 52 and 53 of 2009. This occurred at a time when there was little pandemic influenza circulation and is therefore unlikely to be attributable to pandemic influenza [9]. Other European countries have also developed realtime systems to monitor excess mortality as part of an EU-funded network, EuroMoMo. This network has reported a slight excess in cumulative mortality in young children which could possibly be attributed to pandemic influenza activity [22].

We report that the majority of fatal cases of confirmed pandemic influenza had an underlying risk factor for severe influenza disease. Similar findings have been reported earlier in the UK [3] and the US [23]. The risk factor distribution for pandemic influenza deaths was very similar to that seen for seasonal influenza deaths, except that the latter group had a larger proportion with no reported clinical risk group according to data obtained from death certificates. This discrepancy could be related to under-reporting of clinically relevant material on the death certificate for seasonal influenza, particularly in the older age groups among whom the prevalence of underlying risk conditions is likely to be high. The measures of risk and impact of pandemic influenza were particularly high for those with underlying neurological disease, respiratory disease, immunosuppression and liver disease. This highlights the importance of implementing effective, targeted prevention measures, although a significant minority of younger adults had no underlying risk factor.

A number of points can be raised regarding specific clinical risk groups:

Those with underlying neurological disease were the largest recognised group of deaths with an underlying risk factor. This group had the highest CFR and the largest population attributable fraction. Successfully targeting this group provides potential to reduce overall pandemic deaths. The majority of these cases were children and young adults with neuro-developmental problems such as cerebral palsy or neuro-muscularskeletal problems such as muscular dystrophy. The US Centers for Disease Control and Prevention have reported that $92 \%$ of the children who died with laboratory-confirmed infection and who had an underlying risk factor had neuro-developmental conditions [24]. This population (particularly under 16 years) is not large in the UK and is often clustered in residential special school settings, further increasing their vulnerability. They have been recognised as a group at higher risk of severe disease from seasonal influenza [25]. Our findings highlight the importance of delivery of pandemic influenza $A\left(\mathrm{H}_{1} \mathrm{~N}_{1}\right)$ vaccine to this group. In addition, these individuals should have rapid access 
to antiviral drugs to modify the clinical course of their infection and should be considered for prophylaxis if exposed.

Those with underlying respiratory disease were the second largest group of deaths with an underlying risk factor. However, as this population, which includes people with asthma and chronic obstructive airways disease, is large, the CFR is considerably lower than for other risk factors such as neurological disease. Asthma and other chronic respiratory diseases have long been recognised as important risk factors for severe illness from influenza infection $[26,27]$, and targeting people with these risk factors with vaccination and early antiviral treatment can prevent a significant number of deaths.

Pregnant women were overrepresented amongst fatal cases in the UK compared with the general population and are at increased risk of death. These observations are similar to what has been seen in the US [7], with reports of deaths usually in the third trimester of pregnancy. Deaths amongst pregnant women have also been observed in other countries [6] e.g. South Africa [28]. More recent work from the US has also shown the benefit of early antiviral treatment [8], and these findings reinforce the importance that pregnant women remain a priority group for the UK pandemic influenza $A\left(\mathrm{H}_{1} \mathrm{~N}_{1}\right)$ vaccine programme.

The findings from this paper, and in particular the information from the RRs and population attributable fractions show that the most vulnerable groups for fatal pandemic influenza virus infection are younger adults with chronic neurological, immunosuppressive and respiratory diseases. Assuming causality, the results suggest that many deaths can be prevented if risk groups are targeted early with effective prevention programmes such as pandemic influenza vaccination. The success of such prevention programmes is contingent upon them achieving rapid high coverage.

\section{Acknowledgements}

The authors wish to acknowledge all colleagues throughout the Health Protection Agency Centre for Infections and HPA Local and Regional Services who contributed to data collection, colleagues in the HPA Regional Microbiology Network and Respiratory Virus Reference Unit who undertook laboratory investigation of respiratory samples from suspect cases, colleagues in Health Protection Scotland, National Public Health Service for Wales and Public Health Agency Northern Ireland who undertook investigation and reporting of cases, and finally the medical staff of the Chief Medical Officer in England.

\section{References}

1. Hak E, Verheij TJ, van Essen GA, Lafeber AB, Grobbee DE, Hoes AW. Prognostic factors for influenza-associated hospitalization and death during an epidemic. Epidemiol Infect. 2001;126 (2):261-8
2. Rizzo C, Bella A, Viboud C, Simonsen L, Miller MA, Rota MC et al. Trends for influenza-related deaths during pandemic and epidemic seasons, Italy, 1969-2001. Emerg Infect Dis. 2007;13(5):694-9.

3. Donaldson LJ, Rutter PD, Ellis BM, Greaves FE, Mytton OT, Pebody RG et al. Mortality from pandemic A/H1 12009 influenza in England: public health surveillance study. BMJ. 2009;339:b5213.

4. Kamigaki T, Oshitani H. Epidemiological characteristics and low case fatality rate of pandemic $\left(\mathrm{H}_{1} \mathrm{~N}_{1}\right) 2009$ in Japan. PLoS Curr Influenza. 2009;RRN1139.

5. Presanis A, Lipsitch M, Daniela De Angelis, Swine Flu Investigation Team New York City Department Of Health And Mental Hygiene, Hagy A, Reed C et al. The severity of pandemic $\mathrm{H}_{1} \mathrm{~N}_{1}$ influenza in the United States, April -- July 2009. PLoS Curr Influenza. 2009; RRN1042.

6. Vaillant L, La Ruche G, Tarantola A, Barboza P. Epidemiology of fatal cases associated with pandemic $\mathrm{H}_{1} \mathrm{~N}_{1}$ influenza 2009. Euro Surveill. 2009; 14 (33). pii=19309. Available from: http:// www.eurosurveillance.org/viewarticle.aspx?articleid=19309

7. Jamieson DJ, Honein MA, Rasmussen SA, Williams JL, Swerdlow $\mathrm{DL}$, Biggerstaff MS et al. H1N1 2009 influenza virus infection during pregnancy in the USA. Lancet. 2009; 374(9688):451-8.

8. Louie JK, Acosta M, Jamieson DJ, Honein MA. Severe $2009 \mathrm{H}_{1} \mathrm{~N}_{1}$ influenza in pregnant and postpartum women in California. $\mathrm{N}$ Engl J Med. 2010;362(1):27-35.

9. Health Protection Agency. National weekly influenza report. 1 July 2009 (week 27). Available from: http://www.hpa.org.uk/ web/HPAwebFile/HPAweb_C/1246433639498

10. Chief Medical Officer letter to Clinicians, Department of Health, United Kingdom. 15 October 2009. Available from: http://www. dh.gov.uk/en/Publicationsandstatistics/Lettersandcirculars/ Dearcolleagueletters/DH_107169

11. Gates P, Noakes K, Begum F, Pebody R, Salisbury D. Collection of routine national seasonal influenza vaccine coverage data from GP practices in England using a web-based collection system. Vaccine. 2009;27(48):6669-77.

12. Pebody RG, Begum F, Gates P, Noakes K, Salisbury D. Influenza vaccination coverage in England, 2000-2008. Euro Surveill. 2008;13(51). pii=19074. Available from: http://www. eurosurveillance.org/ViewArticle.aspx?Articleld=19074

13. Birth Statistics 2008. Series FMI No. 37. 9 February 2010. Office for National Statistics, United Kingdom. Available from: http://www.statistics.gov.uk/downloads/theme population/ FM1-37/FM1_37_2008.pdf

14. National Centre for Social Research et al. National Survey of Sexual Attitudes and Lifestyles II, 2000-2001. 2005; Colchester, Essex, UK Data Archive. August 2005. SN: 5223.

15. Health Protection Agency. Method used to estimate new pandemic $\left(\mathrm{H}_{1} \mathrm{~N}_{1}\right) 2009$ influenza cases in England in the week 3 August to 9 August 2009. Available from: http://www.hpa. org.uk/web/HPAwebFile/HPAweb_C/1250150839845

16. Ellis J, Iturriza M, Allen R, Bermingham A, Brown K, Gray J et al. Evaluation of four real-time PCR assays for detection of influenza $A\left(\mathrm{H}_{1} \mathrm{~N}_{1}\right)$ v viruses. Euro Surveill. 2009;14(22). pii=19230. Available from: http://www.eurosurveillance.org/ ViewArticle.aspx?Articleld $=19230$

17. Ellis J, Curran M. Simultaneous Molecular Detection and Confirmation of Influenza $\mathrm{AH}_{5}$, with Internal Control. Stephenson JR, Warnes A, editors. Diagnostic Virology Protocols. Humana Press, 1999.

18. Pitman RJ, Melegaro A, Gelb D, Siddiqui MR, Gay NJ, Edmunds WJ. Assessing the burden of influenza and other respiratory infections in England and Wales. J Infect. 2007;54 (6):530-8.

19. Serum cross-reactive antibody response to a novel influenza $A$ $\left(\mathrm{H}_{1} \mathrm{~N}_{1}\right)$ virus after vaccination with seasonal influenza vaccine. MMWR Morb Mortal Wkly Rep. 2009;58(19):521-4.

20. Miller E, Hoschler K, Hardelid P, Stanford E, Andrews N, Zambon MC. Incidence of 2009 pandemic influenza $\mathrm{A} \mathrm{H}_{1} \mathrm{~N}_{1}$ infection in England: a cross-sectional serological study. Lancet. 2010;375(9720):1100-8.

21. Wilson N, Baker MG. The emerging influenza pandemic: estimating the case fatality ratio. Euro Surveill. 2009;14(26). pii=19255. Available from: http://www.eurosurveillance.org/ ViewArticle. aspx?Articleld $=19255$

22. Mazick A, Gergonne B, Wuillaume F, Danis K, Vantarakis A, Uphoff $\mathrm{H}$ et al. Higher all-cause mortality in children during autumn 2009 compared with the three previous years: pooled results from eight European countries. Euro Surveill. 2010;15(5). pii=19480. Available from: http://www. eurosurveillance.org/ViewArticle.aspx?Articleld=19480 
23. Hospitalized patients with novel influenza $A\left(\mathrm{H}_{1} \mathrm{~N}_{1}\right)$ virus infection - California, April-May, 2009. MMWR Morb Mortal Wkly Rep. 2009;58(19):536-41.

24. Surveillance for pediatric deaths associated with 2009 pandemic influenza $A\left(\mathrm{H}_{1} \mathrm{~N}_{1}\right)$ virus infection - United States, April-August 2009. MMWR Morb Mortal Wkly Rep. 2009;58(34):941-7.

25. Keren R, Zaoutis TE, Bridges CB, Herrera G, Watson BM, Wheeler $A B$ et al. Neurological and neuromuscular disease as a risk factor for respiratory failure in children hospitalized with influenza infection. JAMA. 2005;294(17):2188-94

26. Nicoll A, Ciancio B, Tsolova S, Blank P, Yilmaz C. The scientific basis for offering seasonal influenza immunisation to risk groups in Europe. Euro Surveill. 2008;13(43). pii=19018.

Available from: http://www.eurosurveillance.org/ViewArticle. aspx?Articleld $=19018$

27. Wongsurakiat $P$, Maranetra KN, Wasi C, Kositanont U, Dejsomritrutai W, Charoenratanakul S. Acute respiratory illness in patients with COPD and the effectiveness of influenza vaccination: a randomized controlled study. Chest. 2004;125(6):2011-20.

28. Archer B, Cohen C, Naidoo D, Thomas J, Makunga C, Blumberg $L$ et al. Interim report on pandemic $\mathrm{H}_{1} \mathrm{~N}_{1}$ influenza virus infections in South Africa, April to October 2009: epidemiology and factors associated with fatal cases. Euro Surveill. 2009;14(42). pii=19369. Available from: http://www. eurosurveillance.org/ViewArticle.aspx?Articleld $=19369$ 